\title{
Shoulder Kinematics Is Not Influenced by External Load During Elevation in the Scapular Plane
}

Marcelo P. de Castro-1,2, Daniel Cury Ribeiro-3, Felipe de C. Forte-4,5, Joelly M. de Toledo-6,Daniela Aldabe-3,7 and Jefferson F. Loss- 6

1-University of Porto;

2-Polytechnic Institute of Porto;

3-University of Otago;

4-Worcestershire Acute Hospitals NHS Trust;

5-Coventry University;

6-Universidade Federal do Rio Grande do Sul;

7-Rede Metodista de Educação do Sul-IPA

The current study aimed to compare the shoulder kinematics (3D scapular orientation, scapular angular displacement and scapulohumeral rhythm) of asymptomatic participants under unloaded and loaded conditions during unilateral shoulder elevation in the scapular plane. We used a repeated-measures design with a convenience sample. Eleven male participants with an age range of 21-28 years with no recent history of shoulder injury participated in the study. The participants performed isometric shoulder elevation from a neutral position to approximately 150 degrees of elevation in the scapular plane in intervals of approximately 30 degrees during unloaded and loaded conditions. Shoulder kinematic data were obtained with videogrammetry. During shoulder elevation, the scapula rotated upwardly and externally, and tilted posteriorly. The addition of an external load did not affect 3D scapular orientation, scapular angular displacement, or scapulohumeral rhythm throughout shoulder elevation $(P>.05)$. In clinical practice, clinicians should expect to observe upward and external rotation and posterior tilt of the scapula during their assessments of shoulder elevation. Such behavior was not influenced by an external load normalized to $5 \%$ of body weight when performed in an asymptomatic population.

Keywords: biomechanics, scapula, scapulohumeral rhythm

The combined movement of the glenohumera and scapulothoracic joints is known as scapulohumeral rhythm (SHR). Such integration enables the scapula to provide a stable base for humeral movements, allowing the shoulder to move throughout its range of motion

(ROM). Therefore, scapular motion and stability are essential to the normal function of the shoulder girdle.

Studies of symptomatic populations have demonstrated an association between musculoskeletal shoulder disorders and abnormal scapular movement. Such neuromuscular dysfunction is considered to increase the risk of subacromial impingement. For instance, Caldwell and colleagues reported decreased scapular upward rotation and posterior tilt during upper limb elevation. They also noted that scapular elevation is a typical scapular movement impairment pattern. Their reports are of particular relevance to clinical assessment and rehabilitation of the shoulder, because rehabilitation programs commonly include the analysis and treatment of scapular motor control.

It is accepted that the scapula tends to rotate upward and externally and to tilt posteriorly during unloaded shoulder elevation in the scapular plane. The effect of external load on shoulder kinematics has been investigated during shoulder abduction and shoulder elevation in the scapular plane. The reported findings for shoulder abduction demonstrated different scapular orientations between unloaded and loaded conditions.

For loaded conditions, Kon et al15 found decreased scapular upward rotation at between $35^{\circ}$ and $45^{\circ}$ of shoulder abduction, whereas Forte et al found greater scapular posterior tilt at $60^{\circ}$ and upward rotation at $60^{\circ}$ and $90^{\circ}$ of shoulder abduction. However, the influence of external load on shoulder kinematics during shoulder elevation in the scapular plane is not clear. Michiels and Grevenstein found that an external load ( 1 and $2 \mathrm{~kg}$ ) does not affect the SHR during shoulder elevation in the scapular plane, whereas McQuade and Smidt18 found different SHR in the scapular plane between an unloaded condition and maximum resistive shoulder elevation. Both studies analyzed only the SHR, so it is not known whether there were differences in shoulder kinematics when comparing internal/external scapular rotation and scapular anterior/posterior tilt in unloaded or externally loaded shoulder elevations. Another study investigated the influence of external load on three-dimensional (3D) scapular orientation. Participants maintained a sequence of stationary shoulder positions (ranging from $0^{\circ}$ to $140^{\circ}$ ) and five external load magnitudes (ranging from 0 to $4 \mathrm{~kg}$ ). The results suggested that scapular internal rotation (protraction) 
was affected by external load, while scapular down/upward rotation and anterior/posterior tilt seemed to have a similar pattern during loaded and unloaded conditions. None of these studies normalized external load by body weight or by one maximum repetition.

Therefore, the level of demand that these trials imposed on the scapular and glenohumeral muscles is not clear.

In addition, the abovementioned studies focused on a single shoulder kinematic variable (eg, SHR or scapular orientation).

Physical therapists commonly assess shoulder kinematics during both unilateral and bilateral shoulder elevation in the scapular plane. Many shoulder impairments are caused by micro- and macro-trauma during unilateral movements in standing positions (eg, occupational tasks requiring shoulder abduction or flexion beyond $90^{\circ}$ or sport tasks, such as the tennis serve). However, previous studies have analyzed only bilateral shoulder elevation, and unilateral shoulder kinematics during elevation in the scapular plane has not been described.

Therefore, it is necessary to analyze how unilateral elevation is affected by a load.

Considering the association between shoulder injury and scapular kinematic dysfunction, the use of SHR as an index of quality of movement in the shoulder complex can be of great aid to the early identification of shoulder movement disorders. Assessing shoulder kinematics in healthy individuals can provide useful information for clinical assessment of patients with shoulder musculoskeletal disorders. Such information can be used as a reference and can help clinicians to predict what a patient's shoulder kinematic pattern will be during the transition from an active exercise with only the segment weight (unloaded condition) to a resisted one. Thus, the purpose of our study was to compare the shoulder kinematics of asymptomatic participants during an unloaded condition with an externally loaded condition that was normalized by body weight during unilateral shoulder elevation in the scapular plane. Differences in shoulder kinematics were assessed by comparing 3D scapular orientation, scapular angular displacement, and SHR. Based on previous studies, it was hypothesized that the scapula would show increased upward and external rotation as well as increased posterior tilt during loaded shoulder elevation in the scapular plane.

\section{Methods}

This is a repeated-measure study with a convenience sample. Ethical approval was granted by the ethical committee of the university in which the research was carried out. All participants provided written, informed consent upon agreeing to participate.

\section{Participants}

Fourteen asymptomatic participants were initially involved in the study. To be included in the study, participants had to be physically active, have no shoulder injuries, and be right handed (this was established by asking participants their preferred hand for writing).

We opted to include right-handed participants to optimize data processing time. Participants were excluded from the study if they presented with pain, had a history of injury to the right upper limb during the previous six months, experienced pain during specific clinical joint tests (Hawkins test for subacromial impingement and anterior and posterior apprehension tests for instability), and performed regular physical activity more than three times a week (to avoid a possible influence of physical training on participants performance). Based on these criteria, two left-handed participants and one with a positive response to the instability tests were excluded from the study. Therefore, the sample consisted of eleven male participants who performed physical activity two or three times per week and who were from 21 to 28 years of age. The sample had a mean height of $176 \pm 6 \mathrm{~cm}$, a mean body mass of $74.8 \pm 7.8 \mathrm{~kg}$, and a mean body mass index of $24.2 \pm 2.7$.

\section{Instruments}

Five digital video cameras (JVC GR-DVL9800; $50 \mathrm{~Hz}$ sample frequency), five computers, five spotlights, and five sturdy camera stands were used to record the images.

A Peak Performance v.5.3 3D calibrator (Peak Performance Technologies, Englewood, USA), one manual goniometer, two dumbbells ( 3 and $4 \mathrm{~kg}$ ), one wrist weight

$(0.5 \mathrm{~kg})$, and nineteen reflective markers with a diameter of $1.2 \mathrm{~cm}$ were used. 


\section{Kinematic Data}

Kinematic data were obtained by means of videogrammetry.

This setup allowed the 3D reconstruction of the body segments using the direct linear transformation method (DLT). To describe spatial position, a global coordinate system (GCS) and local coordinate system (LCS) were used according to recommendations from the International Society of Biomechanics. The GCS refers to the environment where data collection was carried out, was used as a reference for the spatial position of the reflective markers, and was established by means of a 3D calibrator. The markers were placed at the following anatomical references: the seventh cervical vertebra, eighth thoracic vertebra, sternum jugular notch, xiphoid process, sternoclavicular joint, acromioclavicular joint, spine of the scapula, inferior angle, acromial angle, coracoid process, glenohumeral rotation center, lateral epicondyle, medial epicondyle, radial styloid process, and ulnar styloid process.

The LCS for the thorax, scapula, and humerus were calculated. The scapula coordinates were calculated in relation to the thorax, and the humerus coordinates were calculated in relation to the scapula. The LCS for the scapula considered $x$ as representative of the posterior/ anterior axis, $y$ as representative of the inferior/superior axis, and $z$ as representative of the medial/lateral axis of the scapula. Hence, scapular movements occurred in the three following directions: (1) The $x$ axis described upward ( - ) and downward $(+)$ rotation; (2) the $y$ axis described external $(-)$ and internal $(+)$ rotation; and (3) the $z$ axis described anterior (-) and posterior (+) tilt.

For kinematic data capture as well as the digitization and reconstruction of images, the videogrammetry system Dvideo v.5.0 (Unicamp, Campinas, Brazil) was used. Intercamera synchronization was performed using the camera's audio band. The data were processed using the one-link segment model applied through Matlab v.7.0 (MathWorks, Massachusetts, USA).

\section{Experimental Protocol}

Firstly, participants' height and weight were measured, and specific clinical joint tests were conducted by an experienced physiotherapist (J.M.T.). Next, the participants stretched their flexor and horizontal adductor shoulder muscles by supporting their forearms (with the elbow and shoulder flexed to $90^{\circ}$ ) on a wall and then turning their bodies to the opposite side of the upper limb that they were stretching. They stretched their extensor and horizontal abductor shoulder muscles by holding one of their upper limbs with the other upper limb at maximal horizontal adduction. Every stretch was held for 30 seconds and was performed bilaterally. Then, the participants warmed up by performing ten repetitions of unloaded shoulder elevations, flexions, and abductions.

Finally, they familiarized themselves with the tasks to be performed.

The order of the data collection for loaded and unloaded conditions was randomized. During loaded conditions, participants held a dumbbell with an external load normalized according to each participant's body mass. During the loaded trials, the external load was equivalent to approximately $5 \%$ of each individual's body mass, and thus ranged from 3 to $4.5 \mathrm{~kg}$. The percentage of body mass was used as load normalization criteria, because load magnitude can represent different efforts among subjects. This load was adopted, because it is frequently applied to exercises during shoulder rehabilitation.

Six shoulder elevation positions in the scapular plane of elevation were recorded for each condition. The scapular plane of elevation was defined as $30^{\circ}$ anterior to the frontal plane. To help each participant keep his upper limb aligned to this plane, a vertically oriented nylon wire was placed near the participant. During each trial, subjects were instructed to elevate their arm in such way that it was immediately adjacent to the nylon wire. The motion in the scapular plane was adopted, since it is commonly used during upper limb musculoskeletal assessment and as a rehabilitation exercise in clinical practice. Starting from resting position with the upper limbs by the side of the body, subsequent positions were set with an interval of approx. $30^{\circ}$ (Figure 1), and the accuracy of the interval was verified with a manual goniometer. The stationary arm of the goniometer was placed perpendicularly to the ground while the mobile arm was positioned along the humeral axis. Video recording lasted no more than five seconds for each position with an interval of at least sixty seconds between recordings to avoid fatigue. The same physiotherapist rearranged all of the scapular markers at the inferior angle, spine of the scapula, coracoid process, and acromioclavicular joint at every position. This method was found to be reliable for analyzing scapular orientation during shoulder abduction, showing a good degree of repeatability for humeral abduction and scapular upward rotation (ICC of 0.98 and 0.83 , respectively). 


\section{Data Analysis}

Three kinematic variables were calculated to assess shoulder kinematics during shoulder elevation with and without external load: scapular orientation, scapular angular displacement, and SHR. Scapular orientation was defined as the 3D scapular position in each of the six positions (internal and external rotation; downward and upward rotation; anterior and posterior tilt) in relation to the thorax; scapular angular displacement was defined as the scapular angular variation between positions; and SHR was defined as the coupled movement between the glenohumeral and scapulothoracic joints and calculated as the ratio between the humeral elevation ROM and scapular upward rotation ROM of each interval between positions.

\section{Statistical Analysis}

To analyze the load's influence on the scapular orientation variable, a three-way repeated-measures ANOVA was conducted with the condition (loaded and unloaded), scapular plane of motion (frontal, transverse, and sagittal) and position of shoulder elevation (positions one to six) as factors. For the scapular angular displacement variable, a three-way repeated-measures ANOVA was also conducted in which the condition (loaded and unloaded), scapular plane of motion (frontal, transverse, and sagittal), and the interval between positions of shoulder elevation (five intervals between positions and the total interval) were the factors. The influence of external load

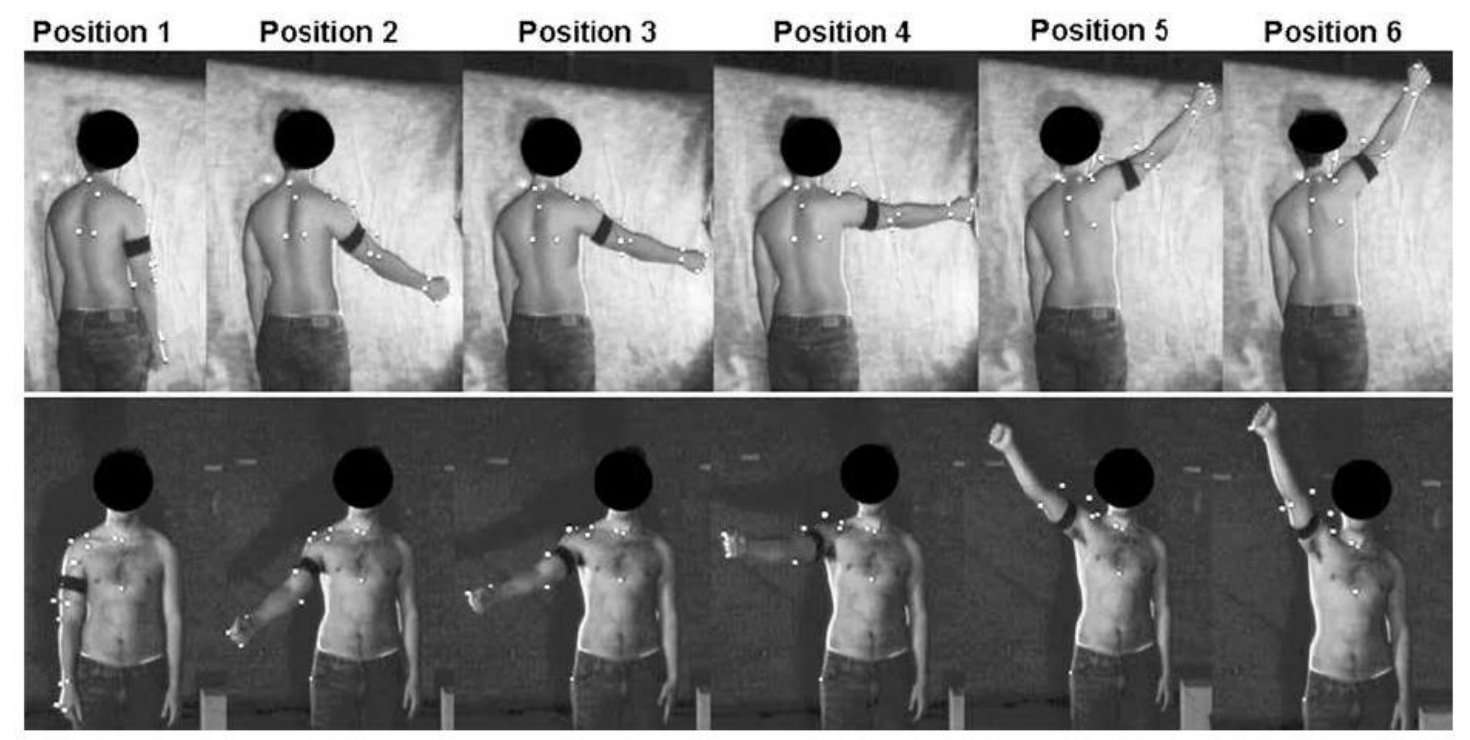

Figure 1 - Image of two of the five digital video cameras during each position.

on the SHR was tested with a two-way repeated-measures ANOVA with the condition (loaded and unloaded) and the interval between positions of shoulder elevation as factors. Considering the ANOVA assumptions, data were found to be normally distributed as indicated by the Shapiro-Wilk test $(P>.05)$. Sphericity for scapular orientation and angular displacement was verified using Mauchly's test. Sphericity for SHR was not found, so a Greenhouse-Geisser correction was used. The purpose of this study required only the interactions among the factors be analyzed. The partial eta square ( $\eta 2 \mathrm{p}$ ) was used to measure the effect sizes, considering that an $\eta 2 p$-value of 0.01 was small, 0.06 was medium, and 0.14 was large. All analyses were performed using the SPSS v.17 software (SPSS Inc, Chicago, USA) with the $\alpha$-value set at 0.05 .

\section{Results}

No differences were found for any of the three dependent variables analyzed (scapular orientation, scapular angular displacement, and SHR). For scapular orientation, the unloaded and loaded conditions were similar for the three scapular planes of motion in all positions of shoulder elevation $(F(10,180)=$ 
1.308; $P=.236 ; \eta 2 \mathrm{p}=.116 ;$ power = .638). The scapular orientation during shoulder elevation showed progressive scapular upward rotation (Figure $2 \mathrm{~A}$ ), a posterior tilt only from position five to the final position (Figure 2B), and a similar scapular internal/ external rotation positioning (Figure 2C).

No statistically significant differences of scapular angular displacement were found between the unloaded and loaded conditions $(F(10,180)=.714 ; P=.709 ; \eta 2 p=.067 ;$ power $=.353)$. Among all intervals analyzed, scapular angular displacement was less than $5^{\circ}$ for both external rotation and scapular tilt movements, whereas scapular upward rotation angular displacement was greater than $5^{\circ}$ for almost all intervals between positions during both conditions (Table 1).

No statistically significant differences were found between unloaded and loaded conditions for SHR ( $F(5$, $60)=.141 ; P=.982 ; \eta 2 \mathrm{p}=.012 ;$ power $=.080)$. Values ranging between two and four were found throughout shoulder elevation in the scapular plane, with the exceptions of the second interval during the unloaded condition $(5.2 \pm 4.8)$ and the fifth interval for the loaded condition $(1.6 \pm 1.0)$. The total SHR for the unloaded condition and externally loaded condition were $3.2 \pm 0.9$ and $2.7 \pm 0.6$, respectively (Table 1).

\section{Discussion}

This study aimed to compare shoulder kinematics between unloaded and loaded shoulder elevation in the scapular plane. Our results showed similar shoulder kinematics (scapular orientation, scapular angular displacement, and SHR) between the two conditions. Therefore, shoulder elevation does not seem to be affected by a provision of external resistance of approximately $5 \%$ of body weight.

To our knowledge, only one study, which was conducted by Pascoal et al, compared 3D scapular orientation between unloaded and loaded conditions during shoulder elevation in the scapular plane. They19 found that load did influence scapular internal/external rotation; however, their report did not clarify whether the external load increased or decreased the internal or external scapular rotation. They also analyzed bilateral shoulder elevation by using five fixed loads (ranging from 0 to $4 \mathrm{~kg}$ ) and by requesting participants to perform shoulder movements 
A UPWARD / DOWNWARD ROTATION

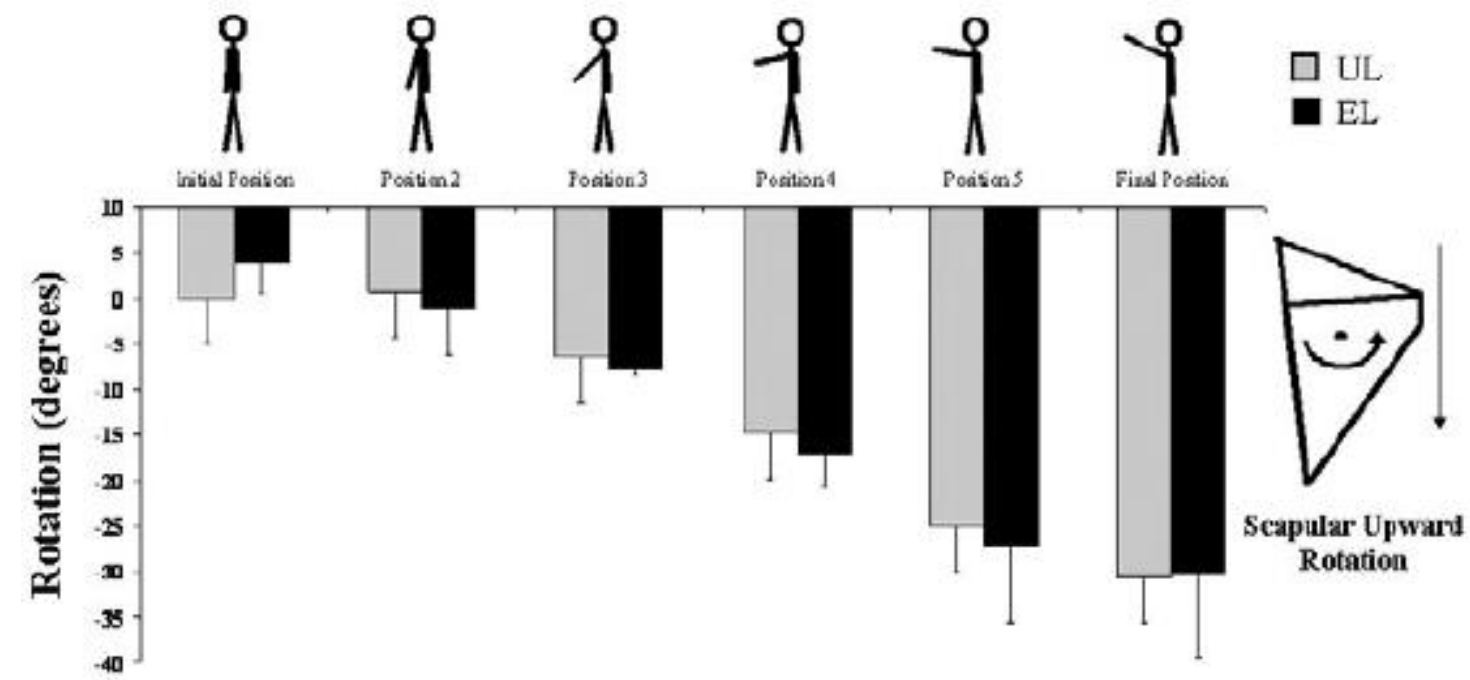

\section{B ANTERIOR/POSTERIOR TILT}

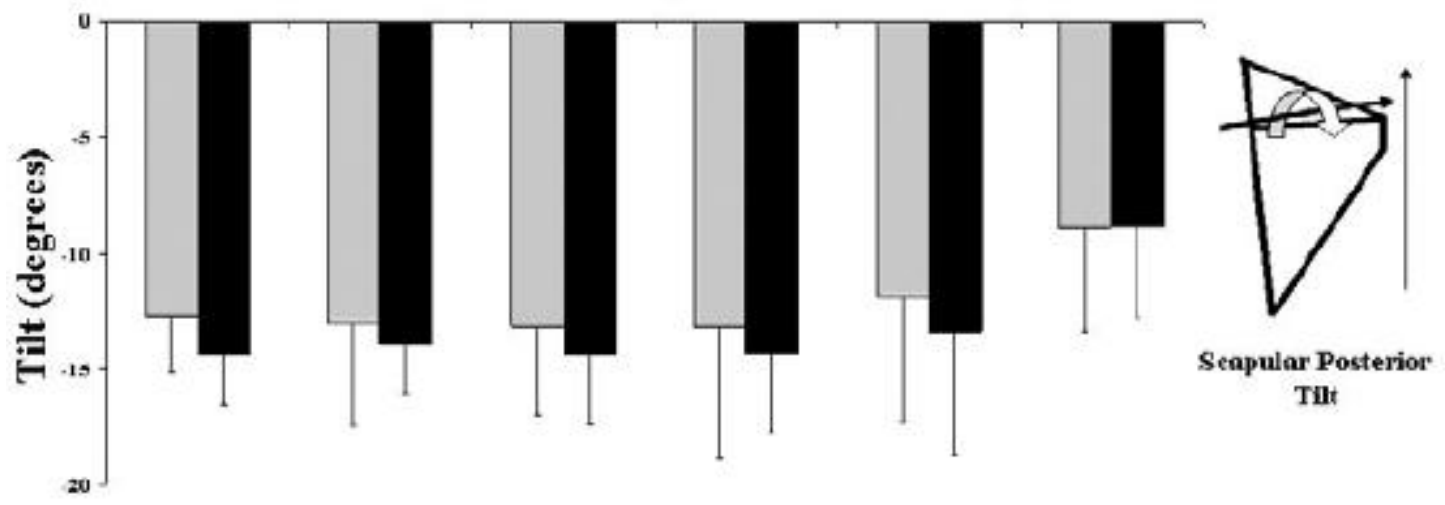

C INTERNAL/EXTERNAL ROTATION

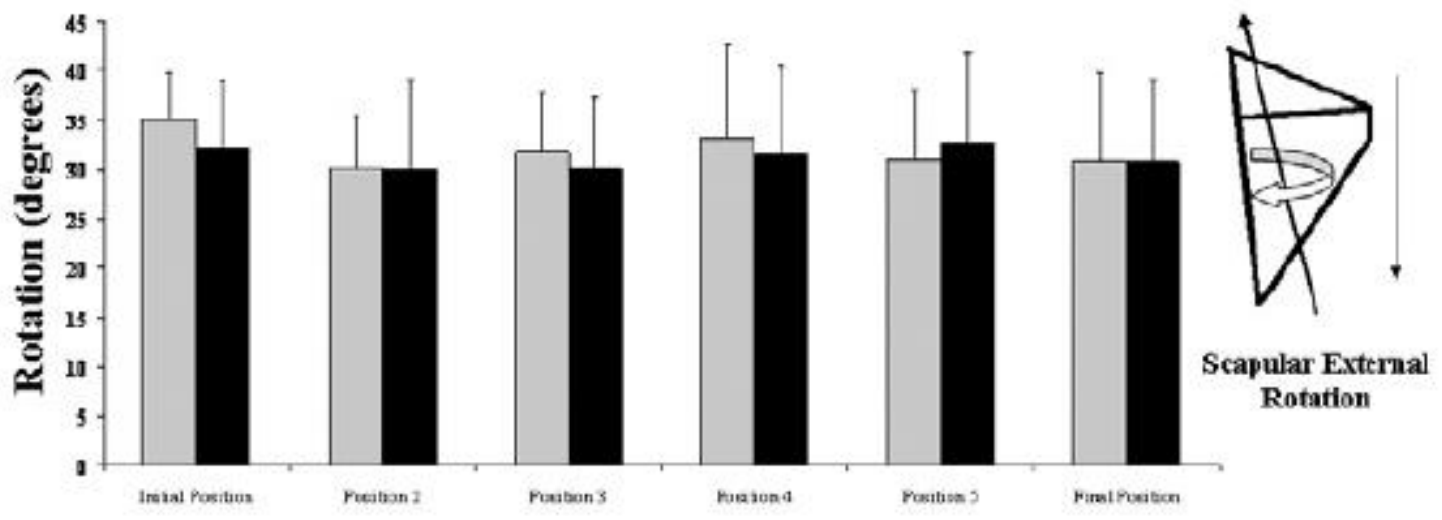

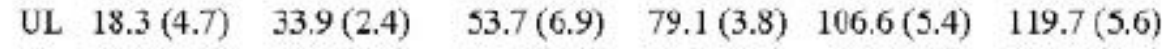

$\begin{array}{lllllll}\text { EL } & 15.1(3.1) & 32.2(5.8) & 54.1(7.1) & 76.1(6.7) & 101(2.8) & 116.2(4.3)\end{array}$

Angle of Elevation (degrees) 
Figure 2 - Mean and standard deviation of the scapular upward / downward rotation (A), anterior/posterior tilt (B) and internal/ external rotation (C). UL—unloaded condition; EL—external loaded condition. The values presented above the $x$-axis title represent the mean and standard deviation for humeral elevation angle for each position.

while seated. In our study, we assessed unilateral shoulder elevation using an external load normalized to body weight, and participants performed shoulder elevation in a standing posture. These differences might have influenced scapular behavior. It is possible that bilateral shoulder elevation leads to different demand levels for trunk stabilization, which would encourage a different scapular orientation pattern.

Previously, our group analyzed 3D scapular orientation during shoulder abduction and found differences between unloaded and externally loaded conditions for posterior tilt at $60^{\circ}$ of shoulder abduction and upward rotation at $60^{\circ}$ and at $90^{\circ}$ of shoulder abduction. Other studies analyzing other variables also indicate differences between shoulder abduction and shoulder elevation in the scapular plane. Toledo et al analyzed the shoulder resultant net moment in both planes of movement performed dynamically with an external load also of $5 \%$ of the body mass. They found a higher external rotator resultant net moment for shoulder abduction in comparison with shoulder elevation in the scapular plane. Moseley et al measured the activity of scapular

Table 1 Mean (SD) values for humeral and scapular angular displacement and scapulohumeral rhythm (SHR)

\begin{tabular}{lccccccc}
\hline ROM Between Positions (Degrees) & $\mathbf{\Delta}$ & $\mathbf{\Delta}$ & $\mathbf{\Delta}$ & $\mathbf{\Delta}$ & $\mathbf{\Delta}$ & $\boldsymbol{\Delta}$ \\
\hline \multirow{2}{*}{ Humeral Elevation } & UL & $13.7 \pm 7.4$ & $21.7 \pm 8.6$ & $22.9 \pm 6.7$ & $30.0 \pm 4.9$ & $11.6 \pm 4.3$ & $99.9 \pm 8.8$ \\
& EL & $13.8 \pm 8.2$ & $23.9 \pm 9.0$ & $22.0 \pm 5.1$ & $26.7 \pm 6.9$ & $13.3 \pm 6.0$ & $99.7 \pm 5.3$ \\
\hline \multirow{2}{*}{ External Rotation } & UL & $-0.7 \pm 2.4$ & $0.0 \pm 1.7$ & $-1.0 \pm 2.5$ & $1.3 \pm 3.2$ & $2.9 \pm 4.4$ & $3.3 \pm 6.0$ \\
& EL & $0.3 \pm 0.8$ & $-1.5 \pm 2.2$ & $0.6 \pm 3.0$ & $1.0 \pm 4.2$ & $2.9 \pm 4.4$ & $4.7 \pm 1.5$ \\
\hline \multirow{2}{*}{ Upward Rotation } & UL & $3.0 \pm 3.4$ & $6.5 \pm 4.3$ & $6.9 \pm 3.4$ & $12.6 \pm 4.1$ & $5.6 \pm 3.8$ & $32.8 \pm 9.2$ \\
& EL & $4.2 \pm 4.7$ & $7.2 \pm 4.3$ & $8.5 \pm 3.7$ & $11.3 \pm 5.3$ & $5.9 \pm 4.6$ & $36.3 \pm 9.6$ \\
\hline \multirow{2}{*}{ Posterior Tilt } & UL & $3.1 \pm 5.5$ & $0.0 \pm 3.9$ & $-3.4 \pm 4.6$ & $2.1 \pm 7.9$ & $1.2 \pm 4.5$ & $3.2 \pm 5.8$ \\
& EL & $2.5 \pm 2.8$ & $-0.1 \pm 4.1$ & $-2.1 \pm 3.1$ & $-1.3 \pm 3.7$ & $1.2 \pm 3.2$ & $1.3 \pm 4.8$ \\
\hline \multirow{2}{*}{ SHR* } & UL & $3.6 \pm 8.1$ & $5.2 \pm 4.8$ & $3.0 \pm 1.8$ & $2.9 \pm 0.7$ & $3.1 \pm 2.0$ & $3.2 \pm 0.9$ \\
& EL & $2.8 \pm 3.1$ & $3.2 \pm 2.4$ & $2.9 \pm 1.3$ & $3.1 \pm 1.7$ & $1.6 \pm 1.0$ & $2.7 \pm 0.6$ \\
\hline
\end{tabular}

Note. ROM, range of motion; UL, unloaded condition; EL, external loaded condition. $\Delta 1$-variation between the positions 1 (rest position) and 2; $\Delta 2$ - variation between the positions 2 and $3 ; \Delta 3$ - variation between the positions 3 and $4 ; \Delta 4$-variation between the positions 4 and $5 ; \Delta 5$ - variation between the positions 5 and 6 (final position); $\Delta$ total — variation between the positions 1 and 6 .

*SHR represents the ratio between the humeral elevation ROM and scapular upward rotation ROM and, thus, it is dimensionless.

muscles with electromyography during 16 exercises that were being used in a particular shoulder rehabilitation program. Among these exercises were shoulder elevations in the scapular plane and shoulder abductions. Moseley et al found differences in the activation patterns between the two aforementioned exercises, and they considered elevation in the scapular plane an optimal exercise for scapular muscle recruitment. Therefore, when elevation in the scapular plane is performed with humeral external rotation (even beyond $90^{\circ}$ of elevation), there might be only minimal impingement of the rotator cuff under the coracoacromial arch, which might help to explain the difference between the present findings and those reported previously for shoulder abduction. Thus, the increased scapular upward rotation and posterior tilt found during the loaded condition while performing shoulder abduction 16 could be one way of minimizing the risk of subacromial impingement.

Regarding SHR, our findings corroborate those by Michiels and Grevenstein, because they found no difference for SHR during shoulder elevation between unloaded and loaded conditions (1 kg and $2 \mathrm{~kg}$ ). These authors used a fixed, external load, whereas we normalized the external load by making it a percentage of each participant's body weight. McQuade and Smidt, on the other hand, found differences in SHR when comparing unloaded and maximal externally loaded conditions during shoulder elevation in the scapular plane. Their results showed a SHR equivalent to 1.8 for the loaded condition for the first $20 \%$ of ROM and a SHR of 3.2 for the unloaded condition. For the other intervals (between 20 and $100 \%$ of ROM), no differences between conditions were found. In the current study, no differences were found for SHR between the loaded and unloaded conditions. Moreover, for our first interval of ROM, the SHR was 2.8 and 3.6 for loaded and unloaded conditions, respectively. One possible explanation for the 
discrepancy between the present findings and those from McQuade and Smidt might be the external load magnitude. We opted to normalize the external load as $5 \%$ of body mass, while McQuade and Smidt used a maximal load.

In view of our results, the behavior of 3D scapular orientation, scapular angular displacement, and SHR during unilateral shoulder elevation in the scapular plane in asymptomatic subjects who are in a standing position is similar between unloaded and loaded conditions.

Therefore, even the scapular movement pattern during unloaded conditions could be used as a reference for resisted shoulder elevation. Each individual's capacity for maintaining a similar scapular movement pattern when performing loaded elevation in the scapular plane could be used as the criterion for progression of shoulderresisted exercises. However, detecting small differences in the scapular orientation, scapular angular displacement, or SHR might be difficult in clinical practice.

Previous studies have questioned the ability of clinicians to examine reliably scapular kinematics through observation.

The clinical value of our results is yet unknown and difficult to interpret. Nevertheless, we recommend our data be read as an indication that loads of $5 \%$ of the body weight do not influence shoulder kinematics during elevation in the scapular plane when performed by healthy, right-handed young males who perform physical activity as mere leisure (two or three times per week). Changes in scapular movement pattern might indicate disrupted scapular neuro-motor control and might increase the risk of shoulder injury. Different shoulder kinematic behaviors have been reported for symptomatic subjects. Ogston and Ludewig9 showed reduced scapular upward rotation (especially at the final range of motion for shoulder elevation) and increased scapular internal rotation in patients with multidirectional shoulder instability. Matias and Pascoal analyzed a similar population (ie, patients presenting with multidirectional shoulder instability), and found excessive internal rotation and delayed posterior tilt. Therefore, risk of injury might be minimized by ensuring that patients have the ability to maintain the observed kinematic pattern while performing resisted shoulder elevation in the scapular plane.

Electromagnetic tracking systems are commonly used for monitoring scapular kinematics.

The accuracy of electromagnetic systems has been assessed to be equal to $0.50^{\circ}$ (root mean square) with a $95 \%$ confidence interval smaller than $4^{\circ}$ for orientation measurements and approximately $20 \mathrm{~mm}$ for linear measurements. The current study used the same videogrammetry system and a similar camera setup as did a previous study that found the linear accuracy of this setup to be equal to $1.7 \mathrm{~mm}$. Melton et al used videogrammetry to present a standard error of measurement of $3^{\circ}$ for the range of motion analysis during shoulder elevation. To our knowledge, specific data regarding accuracy of scapular measurements using a videogrammetry system have not been described in the literature.

Thus, the comparison of results from studies using different methods to monitor scapular movement should be performed carefully. Previous studies have assessed scapular movements by analyzing a sequence of static position (or quasi-static analysis, as dubbed by another study). This method might involve a different movement pattern of scapular muscles, because it requires participants are required to sustain an isometric position; however, there is only a small error associated with the markers' replacement. Therefore, this is an accepted method for monitoring scapular movements, and it is considered to reflect the scapular orientation changes.

Scapular motion analysis has been assessed using different approaches. Brochard et al compared the accuracy and intertrial reliability of three methods for the assessment of scapular orientation by using reflective markers and stereo-photogrammetry: three markers placed on three landmarks of the scapula; a rigid acromion marker cluster; and three markers fixed on the skin of the acromion. The first method resulted in significant error measurements, whereas the two acromion marker methods improved accuracy for several scapular rotations but resulted in large errors for other rotations. Brochard et al analyzed a sequence of stationary positions with the rearrangement of the scapular markers, and compared them to other sequences of stationary positions without rearranging the scapular markers after calibration based on initial and final scapular orientation. These authors found that this calibration method allowed for accurate and reliable measures of scapular kinematics. Among the currently practiced noninvasive methods, palpation (eg, rearranging scapular markers) is believed to be the standard and a reliable method for measuring scapular motion. The current study assessed scapular orientation based on the palpation method, and, since it has been considered the gold standard for other studies, the internal consistency of the present method can be considered reliable.

This study has some limitations. The influence of skin and subcutaneous tissue over the movement of reflective markers could be a source of limitation. However, we believe we have mitigated this effect by repositioning the scapular markers for each position. In addition, the determination of the bony 
landmarks could influence inverse kinematic results. De Groot performed a methodological study for quantifying the sources of variability for measuring 3D shoulder movement, and concluded that the palpation method is accurate for 3D orientation of the shoulder mechanism and for intraindividual studies, because the method presented a palpation error of approximately $2^{\circ}$ in the inverse kinematic results.

In the current study, the effect size of the scapular position and scapular angular displacement (dependent variables) was medium, and it was small for the SHR. Thus, it is plausible to assume that a Type II error may have occurred. To achieve $80 \%$ statistical power, it would be necessary to collect six more subjects for the scapular position, 19 more subjects for the scapular angular displacement, and an additional of 153 subjects for the SHR.

Finally, the reliability analysis of the method used in the current study was performed in the frontal plane with only four subjects. Furthermore, no measurements that could provide information regarding intraindividual variability of the current experimental protocol were made.

In conclusion, the results of the current study suggest that external load (equivalent to $5 \%$ of the body mass) does not influence 3D scapular orientation, scapular angular displacement, or SHR during unilateral shoulder elevation in the scapular plane. When investigating asymptomatic nonathletic young males with right upper limb dominance, no differences between loaded and unloaded conditions should be expected.

\section{References}

1. McClure PW, Michener LA, Sennett BJ, Karduna AR. Direct 3-dimensional measurement of scapular kinematics during dynamic movements in vivo. J Shoulder Elbow Surg. 2001;10:269-277. PubMed doi:10.1067/mse.2001.112954

2. Kebaetse M, McClure P, Pratt NA. Thoracic position effect on shoulder range of motion, strength, and three-dimensional scapular kinematics. Arch Phys Med Rehabil. 1999;80:945-950. PubMed doi:10.1016/S0003-9993(99)90088-6

3. Ebaugh DD, McClure PW, Karduna AR. Three-dimensional scapulothoracic motion during active and passive arm elevation. Clin Biomech (Bristol, Avon). 2005;20:700- 709. PubMed doi:10.1016/j.clinbiomech.2005.03.008

4. Kibler WB, McMullen J. Scapular dyskinesis and its relation to shoulder pain. J Am Acad Orthop Surg. 2003;11:142-151. PubMed

5. McClure PW, Michener LA, Karduna AR. Shoulder function and 3-dimensional scapular kinematics in people with and without shoulder impingement syndrome. Phys Ther. 2006;86:1075-1090. PubMed

6. Hebert L, Moffet H, McFadyen BJ, Dionne CE. Scapular behavior in shoulder impingement syndrome. Arch Phys Med Rehabil. 2002;83:60-69. PubMed doi:10.1053/ apmr.2002.27471

7. Lin JJ, Lim HK, Soto-quijano DA, et al. Altered patterns of muscle activation during performance of four functional tasks in patients with shoulder disorders: interpretation from voluntary response index. J Electromyogr Kinesiol. 2006;16:458-468. PubMed doi:10.1016/j.jelekin.2005.09.008

8. Lin JJ, Lim HK, Yang JL. Effect of shoulder tightness on glenohumeral translation, scapular kinematics, and scapulohumeral rhythm in subjects with stiff shoulders. J Orthop Res. 2006;24:1044-1051. PubMed doi:10.1002/jor.20126

9. Ogston JB, Ludewig PM. Differences in 3-dimensional shoulder kinematics between persons with multidirectional instability and asymptomatic controls. Am J Sports Med. 2007;35:1361-1370. PubMed doi:10.1177/0363546507300820

10. Caldwell C, Sahrmann S, Van Dillen L. Use of a movement system impairment diagnosis for physical therapy in the management of a patient with shoulder pain. J Orthop Sports Phys Ther. 2007;37:551-563. PubMed doi:10.2519/jospt.2007.2283

11. Magarey ME, Jones MA. Dynamic evaluation and early management of altered motor control around the shoulder complex. Man Ther. 2003;8:195-206. PubMed doi:10.1016/S1356-689X(03)00094-8

12. Ludewig P, Cook T, Nawoczenski D. Three-dimensional scapular orientation and muscle activity at selected positions of humeral elevation. J Orthop Sports Phys Ther. 1996;24:57-65. PubMed doi:10.2519/jospt.1996. 24.2.57

13. Ludewig P, Phadke V, Braman J, Hassett D, Cieminski C, LaPrade R. Motion of the shoulder complex during multiplanar humeral elevation. J Bone Joint Surg Am. 2009;91:378-389. PubMed doi:10.2106/JBJS.G.01483

14. Meyer K, Saether E, Soiney E, Shebeck M, Paddock K, Ludewig P. Three-dimensional scapular kinematics during the throwing motion. J Appl Biomech. 2008;24:24-34. PubMed 
15. Kon Y, Nishinaka N, Gamada K, Tsutsui H, Banks S. The influence of handheld weight on the scapulohumeral rhythm. J Shoulder Elbow Surg. 2008;17:943-946.PubMed doi:10.1016/j.jse.2008.05.047 16. Forte F, Castro M, Toledo J, Ribeiro D, Loss J. Scapular kinematics and scapulohumeral rhythm during resisted shoulder abduction - Implications for clinical practice. Phys Ther Sport. 2009;10:105-111. PubMed doi:10.1016/j.ptsp.2009.05.005

17. Michiels I, Grevenstein J. Kinematics of shoulder abduction in the scapular plane. On the influence of abduction velocity and external load. Clin Biomech (Bristol, Avon). 1995;10:137-143. PubMed doi:10.1016/0268-0033(95)93703-V

18. McQuade KJ, Smidt GL. Dynamic scapulohumeral rhythm: the effects of external resistance during elevation of the arm in the scapular plane. J Orthop Sports Phys Ther. 1998;27:125-133. PubMed doi:10.2519/jospt.1998.27.2.125

19. Pascoal AG, van der Helm FF, Correia P, Carita I. Effects of different arm external loads on the scapulohumeral rhythm. Clin Biomech (Bristol, Avon). 2000;15(Suppl 1):S21-S24. PubMed doi:10.1016/S02680033(00)00055-3

20. Sahrmann S. Diagnosis and Treatment of Movement Impairment Syndromes. 1st ed. St. Louis: Mosby; 2001.

21. McQuade KJ, Dawson J, Smidt GL. Scapulothoracic muscle fatigue associated with alterations in scapulohumeral rhythm kinematics during maximum resistive shoulder elevation. J Orthop Sports Phys Ther. 1998;28:74-80. PubMed doi:10.2519/jospt.1998. 28.2.74

22. Incel NA, Ceceli E, Durukan PB, Erdem HR, Yorgancioglu ZR. Grip strength: effect of hand dominance. Singapore Med J. 2002;43:234-237. PubMed

23. Tzannes A, Murrell GA. Clinical examination of the unstable shoulder. Sports Med. 2002;32:447-457. PubMed doi:10.2165/00007256-200232070-00004

24. Abdel-Aziz YI, Karara HM. Direct linear transformation from comparator coordinates into object space coordinates in close-range photogrammetry. ASP Symposium on Close-Range photogrammetry. 1971:118.

25. Winter DA. Biomechanics and Motor Control of Human Movement. 3 ed. New Jersey: John Wiley \& Sons Ltd.; 2005.

26. Wu G, van der Helm FC, Veeger HE, et al. ISB recommendation on definitions of joint coordinate systems of various joints for the reporting of human joint motion-Part II: shoulder, elbow, wrist and hand. J Biomech. 2005;38:981- 992. PubMed doi:10.1016/j.jbiomech.2004.05.042

27. Figueroa PJ, Leite NJ, Barros RM. A flexible software for tracking of markers used in human motion analysis. Comput Methods Programs Biomed. 2003;72:155-165. PubMed doi:10.1016/S01692607(02)00122-0

28. Leite de Barros RM, Russomanno TG, Brenzikover R, Figueroa PJ. A method to synchronise video cameras using the audio band. J Biomech. 2006;39:776-780. PubMed doi:10.1016/j.jbiomech.2004.12.025

29. Ribeiro DC, Loss JF. Assessment of the propagation of uncertainty on link segment model results. Motor Control. 2010;14:411-423. PubMed

30. Nevill AM, Ramsbottom R, Williams C. Scaling physiological measurements for individuals of different body size. Eur J Appl Physiol. 1992;65:110-117. PubMed doi:10.1007/BF00705066

31. Stevens J. Applied multivariate statistics for the social sciences. 4th ed. Mahwah, NJ: Lawrence Erlbaum Associates; 2002.

32. de Toledo JM, Ribeiro DC, de Castro MP, et al. Comparison of shoulder resultant net moment between three different exercises and load conditions. Physiother Theory Pract. 2013;29:124-132. PubMed doi:10.3109/09593985.2012.699606

33. Moseley JB, Jobe FW, Pink M, Perry J, Tibone J. EMG analysis of the scapular muscles during a shoulder rehabilitation program. Am J Sports Med. 1992;20:128-134. PubMed doi:10.1177/036354659202000206 34. Hickey BW, Milosavljevic S, Bell ML, Milburn PD. Accuracy and reliability of observational motion analysis in identifying shoulder symptoms. Man Ther. 2007;12:263- 270 . PubMed doi:10.1016/j.math.2006.05.005

35. Matias R, Pascoal A. The unstable shoulder in arm elevation: a three-dimensional and electromyographic study in subjects with glenohumeral instability. Clin Biomech (Bristol, Avon). 2006;21(Suppl 1):S52-S58. PubMed doi:10.1016/j.clinbiomech.2005.09.014

36. Meskers CG, Koppe PA, Konijnenbelt MH, Veeger DH, Janssen TW. Kinematic alterations in the ipsilateral shoul der of patients with hemiplegia due to stroke. Am J Phys Med Rehabil. 2005;84:97-105. PubMed doi:10.1097/01. PHM.0000150792.26793.E9 
37. Borstad JD, Ludewig PM. The effect of long versus short pectoralis minor resting length on scapular kinematics in healthy individuals. J Orthop Sports Phys Ther. 2005;35:227-238. PubMed doi:10.2519/ jospt.2005.35.4.227

38. Ludewig PM, Cook TM. Alterations in Shoulder Kinematics and Associated Muscle Activity in People With Symptoms of Shoulder Impingement. Phys Ther.2000;80:276-291. PubMed

39. Johnson MP, McClure PW, Karduna AR. New method to assess scapular upward rotation in subjects with shoulder pathology. J Orthop Sports Phys Ther. 2001;31:81-89. PubMed doi:10.2519/jospt.2001.31.2.81

40. Karduna AR, McClure PW, Michener LA. Scapular kinematics: effects of altering the Euler angle sequence of rotations. J Biomech. 2000;33:1063-1068. PubMed doi:10.1016/S0021-9290(00)00078-6

41. McClure PW, Bialker J, Neff N, Williams G, Karduna A. Shoulder function and 3-dimensional kinematics in people with shoulder impingement syndrome before and after a 6-week exercise program. Phys Ther. 2004;84:832-848. PubMed

42. Barnett ND, Duncan RDD, Johnson GR. The measurement of three dimensional scapulohumeral kinematics - a study of reliability. Clin Biomech (Bristol, Avon). 1999;14:287- 290. PubMed doi:10.1016/S0268-0033(98)00106-5

43. Melton C, Mullineaux DR, Mattacola CG, Mair SD, Uhl TL. Reliability of video motion-analysis systems to measure amplitude and velocity of shoulder elevation. J Sport Rehabil. 2011;20:393-405. PubMed

44. van Andel C, van Hutten K, Eversdijk M, Veeger D, Harlaar J. Recording scapular motion using an acromion marker cluster. Gait Posture. 2009;29:123-128. PubMed doi:10.1016/j.gaitpost.2008.07.012

45. Jones L, Holt C, Bowers A. Movement of the Shoulder Complex - The development of a measurement technique based on proposed ISB standards. Paper presented at: The Ninth International Symposium on 3D Analyses of Human Movement 2006; Valenciennes, France.

46. Brochard S, Lempereur M, Rémy-Néris O. Accuracy and reliability of three methods of recording scapular motion using reflective skin markers. Proc Inst Mech Eng H. 2011;225:100-105. PubMed doi:10.1243/09544119JEIM830

47. Brochard S, Lempereur M, Rémy-Néris O. Double calibration: An accurate, reliable and easy-to-use method for 3D scapular motion analysis. J Biomech. 2011;44:751-754. PubMed doi:10.1016/j.jbiomech.2010.11.017

48. van der Helm FC, Veeger HE. Quasi-static analysis of muscle forces in the shoulder mechanism during wheelchair propulsion. J Biomech. 1996;29:39-52. PubMed doi:10.1016/0021-9290(95)00026-7

49. Meskers CG, Vermeulen HM, de Groot JH, van Der Helm FC, Rozing PM. 3D shoulder position measurements using a six-degree-of-freedom electromagnetic tracking device. Clin Biomech (Bristol, Avon). 1998;13:280-292. PubMed doi:10.1016/S0268-0033(98)00095-3

50. de Groot JH. The scapulo-humeral rhythm: effects of 2-D roentgen projection. Clin Biomech (Bristol, Avon). 1999;14:63-68. PubMed doi:10.1016/S0268-0033(98)00027-8

51. de Groot JH. The variability of shoulder motions recorded by means of palpation. Clin Biomech (Bristol, Avon). 1997;12:461-472. PubMed doi:10.1016/S0268-0033(97)00031-4 